\title{
The Research on OA Based On Mobile Telephone
}

\author{
Tao Li and Lirong Qiu \\ 1. School of information and electrical engineering, Ludong University \\ Yantai, China \\ 2. School of Information Engineering, Minzu University of China \\ Beijing, China \\ litao_888@sina.com,qiu_lirong@126.com
}

\begin{abstract}
The global trend of the information and network has changed the peoples' life and work style. The traditional OA model can't keep step with the rapid development of the information society. Time and space becomes a main obstacle to the OA. This paper puts forwards a new thought to solve this problem by designing a mobile message module. It can realize mobile OA and may promote the development of the E-government.
\end{abstract}

Keywords: Mobile OA model, GSM, E-government

\section{Introduction}

With the development and popularization of the information technology, network technology especially with the development of Internet, the problems include the standard and cost of information sharing and transmission has been solved. The settlement of these problems make it possible to let information becomes the basic of the dominate decision-making. Mobile OA rises at the historic moment. It is an information assistant tool and it can search information that is needed by users through Internet to process the decision in time. It provides a good platform for publics who can't acquire the government information and policies because the limited time and space. It can also become a mobile OA model and is part of the OA functions. Its main design thought is to provide services for users by the SMS sending equipment when users are off line. It can send the same information to many users at one time by the block sending function. In this way, it can save time and can promote the government efficiency and it also adept to modern society.

\section{The Concept of E-government}

The electronic government affairs are the government apparatus applying modern information and the communication, will manage and the service carries on the integration through the network technology, will realize the official organization structure and the work flow optimized reorganization on Internet, between the surmounting time and spatial and department's separation limit, will provide high quality and omni-directional, the standard to the society but is transparent, conforms to the 
international standard management and the service. In other words, it is the use of IT and e-commerce to provide access to government information and delivery ${ }^{[5]}$ of public services to citizens and business partners.

The first action plan on e-government in 1999 established a three-stranded approach to online delivery. All government services are at one of these stages of evolution. While some are progressing faster than others, all will traverse these three phases:

\section{(1) The Information Services}

This requires that all public service information should be placed online through the websites of agencies and departments. The government generates huge volumes of information every day and not all of them are immediately accessible. The Internet and the ICTs will bring all of the value information together and allow for its publication. This first step in e-government is important, even to the citizen that is not IT literate or web enabled, as it means that the information is available and can be seen and read.

\section{(2) The Interactive services}

The next step is the delivery of public services online, enabling complete transactions to be conducted through the electronic channel. The key aspect of this phase is the interaction between the citizens and government. This may start with basic functions such as email contact information or feedback forms that allow for comments.

\section{(3) The Integrated Services}

The final phase is the alignment of services and information around citizen needs in an integrated manner through a single point of contact with government.

The development of the Internet technology and the increasing require of people compel the development of the e-government. The government has made grate progress in integrating IT investments across agencies around groups of citizens. Much of the improvement has been accomplished through the deployment of the E-Government projects. The projects are organized into "portfolios" or common groupings, defined by the customer segment served. Following is a description of each portfolio, including three goals:

Government to Citizen (G2C): The objective of the $\mathrm{G} 2 \mathrm{C}$ portfolio is to provide one-stop, on-line access to information and services to individuals. Citizens should be able to find what they need quickly and easily, and access information in minutes or seconds, instead of days or hours. For example, the GovBenefits.gov portal is expanding to provide potential beneficiaries with instant access to information for all government benefit programs and services through a single web site.

Government to Business (G2B): The goals of the G2B portfolio are to reduce burdens on business, provide one-stop access to information and enable digital communication using the language of e-business (for example, XML). The Federal government should not continue to make businesses report the same data, multiple times to multiple agencies. The government should reuse the data reported appropriately and take advantage of commercial electronic transaction protocols. The deployment of more effective 
technologies will help streamline the myriad of reporting requirements as well as facilitate a more efficient means for businesses to interact with the government. For example, with the implementation of the E-Rulemaking initiative, businesses will no longer need the assistance of a lawyer or lobbyist to participate in the regulatory process.

Government to Government (G2G): The G2G portfolio's primary goal is to enable federal, state and local governments to more easily work together to better serve citizens within key lines of business. To achieve this goal the Federal government must make it easier for states and localities to meet reporting requirements, while promoting the use of performance measurements. State and local governments will see significant administrative savings and will be able to improve program delivery because the data necessary to measure performance will be more accurate and timely. Improving the way that information is shared among all levels of government has numerous benefits. For example, one homeland security initiative is a secure portal that will improve the disaster management process by simplifying and unifying the interaction between Federal, state, and local public safety personnel.

\section{The Basic Design Thought of This System}

Although the construction of E-government promotes the government efficiency and let publics acquire exact information in time. It has its limits:

(1) Because the traditional E-government system is based on $\mathrm{B} / \mathrm{S}$ mode, if the publics have no computer, they can't receive information.

(2) The transmit speed is affected by network speed, and the information may be delayed because of the disconnection of network.

(3) Data may be lost when some unexpected affair happened in the process of information transmit.

(4) Users may be unable to receive information when they are not on line.

(5) Virus is not a slighting problem, we cannot guarantee information is stole in the information transmit process.

The above localizations lead to baffles when the publics receive information. It seriously affected the electronic government affairs' normal work, and becomes the bottleneck between government information and public information.

E-government is consisted by inner network and outer network. The main function of the inner network is to deal with the inner information of the government that includes files transmission, files sharing and e-mails etc. The main function of outer network is to transmit and feed back the information. The main design thought of this system is to watch the outer database in every time through a system service course. If there is no new information, the system will enter into a dormancy status. Once new information comes in, it will deliver the information to GSM/GPRS system. It sends the new information to mobile telephone by broadcast through the mobile telephone SMS service platform. In this way, publics can acquire and feedback information through the SMS platform and send them to inner network. Thus, it can realize the communication between publics and 
government to promote the government efficiency. The system can solve the database abnormity, sever abnormity and GSM/GPRS abnormity etc. So it assures the normal sending of the SMS. Even if users turn off the mobile phone, they also can receive the information when they open it. The structure of the system is shown in Figure 1.

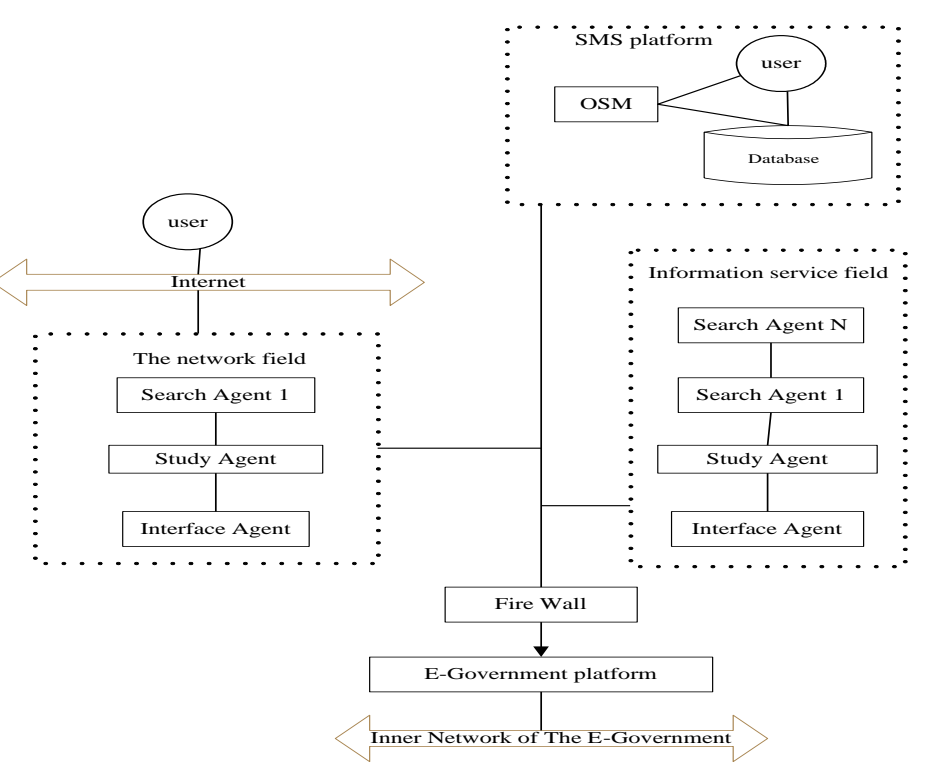

Figure 1. The System Structure

\section{The Work Flow of This System}

Database is first connected in the course of sending by using mobile phone. If database is connected, then data will be read, otherwise database will be connected again until database is successfully connected. Then, the port will be open to code SMS. Then, to judge if Modem is on line, if it is not on line, connect it. At last, to judge if the phone is open, if open, send the information to the phone directly, or store it for later use. The system judges if the phone is open at fixed time until the information is send successfully. The work flow is shown in Figure 2. 


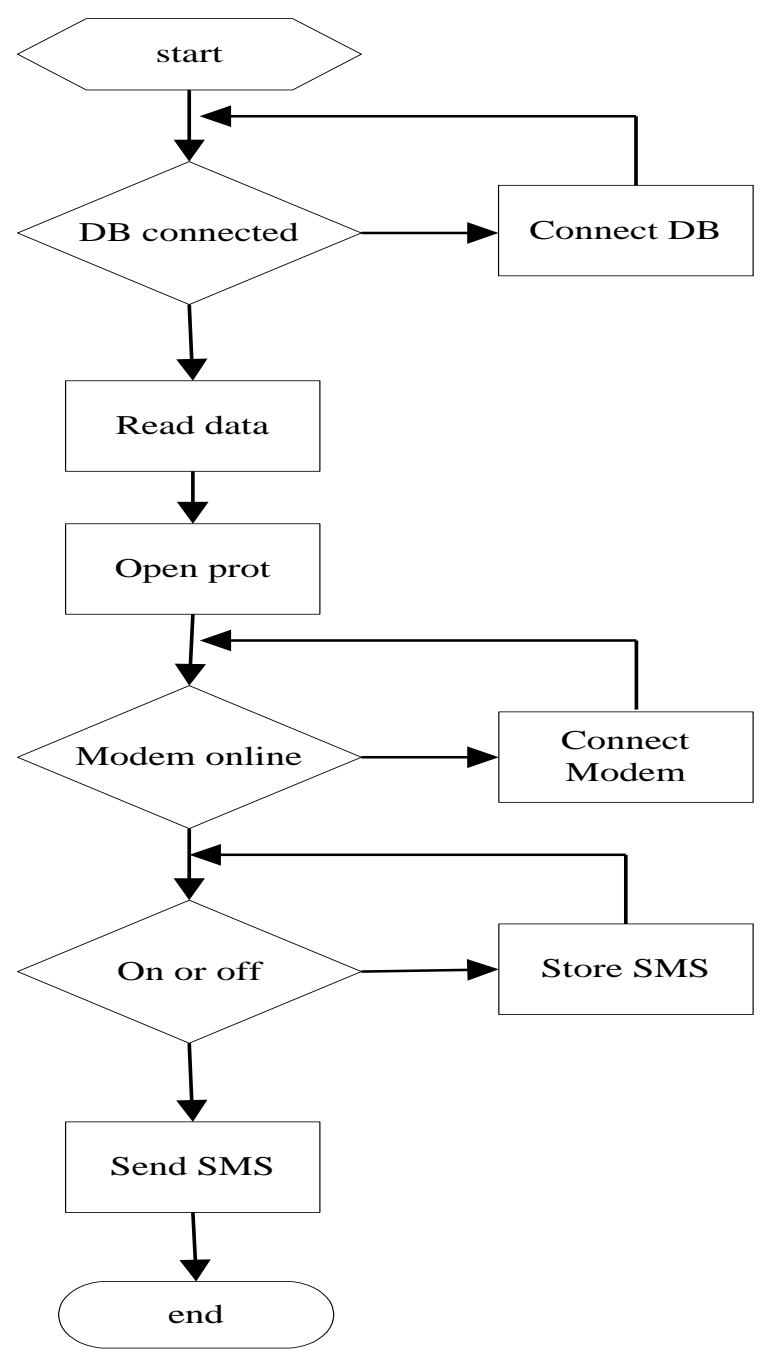

Figure 2. The Work Flow of This System

\section{The Key Technology of this System}

\subsection{Sending Chinese SMS by GSM MODEM}

The function of GSM SMS model is to transmit data, so it may use the phone number when it is used. Six or eight number in data wrap presents the phone number. The low four numbers and the high four numbers in the data byte present one decimal number through BCD code. In this way, each byte can present two phone numbers, and six bytes will present twelve phone numbers. Because phone numbers in our country is changed into eleven phone numbers, so we must add zero behind it to form twelve numbers. The detailed steps: (1) plus zero into the phone number; (2) divide two numbers into one group from left to right; (3) change each group into BCD code. If we need to revert the data in data wrap into the phone numbers, the steps are on the other way round.

There are three modes to send and receive SMS by GSM MODEM: the block mode, the text mode and Pdu mode. We haven't found any factories to claim holding this the block mode. The Pdu mode is difficult to exploit and it is propagated because it need 
compile expert function to convert the Pdu mode. Comparatively, it is easy and convenient to apply the text mode, so this is not a bad choice. But the text mode can only send ASCII code and can't send Chinese Unicode ${ }^{[5]}$.

At first, add Ms Comm control tool in the project. If there is no control tool, you should right click the component column, choose "component" or choose "component" in "project" menu and then choose Microsoft Comm Control 6.0. After add the MS Comm control tool, we can set up some parameters. If we use Nokia mobile phone, we must install data suite, then in comport of the MS Comm we choose 3; if we use other mobile phone and use infrared ray port, we must install infrared ray driving program. Simple SMS can exploit perfect and abundant applied programs such as sending and receiving SMS and dial-up etc according to AT instruction sets with VB cluster ports.

\subsection{The Use of MS COMM}

There are two means to exploit cluster ports using Delphi, the first one is to use API function in Windows, the other one is to adopt MS Comm control tool using Microsoft. It is difficult to compile cluster ports programs using API because we need master communication knowledge. The merits of it are: (1) the functions are mightiness; (2) the application fields are wide; (3) it is fit for compile difficult lower level programs. It is much simple to use MS Comm control tool, this control tool has many properties and affairs related with cluster ports ${ }^{[6]}$.

\subsection{SMS Code}

The SMS contents are converted into Unicode 16, and we give its converting arithmetic.

(1) Coding arithmetic:

Function EncodeWideString(Value:WideString):String;

//encode the widestring to saveable string

var

I: Integer;

begin

Result:=";

for $\mathrm{I}:=1$ to Length(Value) do begin

Result:=Result+IntToHex(WORD(Value[I]) shr 8,2);

Result:=Result+IntToHex(WORD(Value[I]) and \$00ff,2);

end;

end;

(2) De-coding arithmetic:

Function DecodeWideString(Value:String):WideString;

//decode the string to widestring

var

I: Integer;

begin 


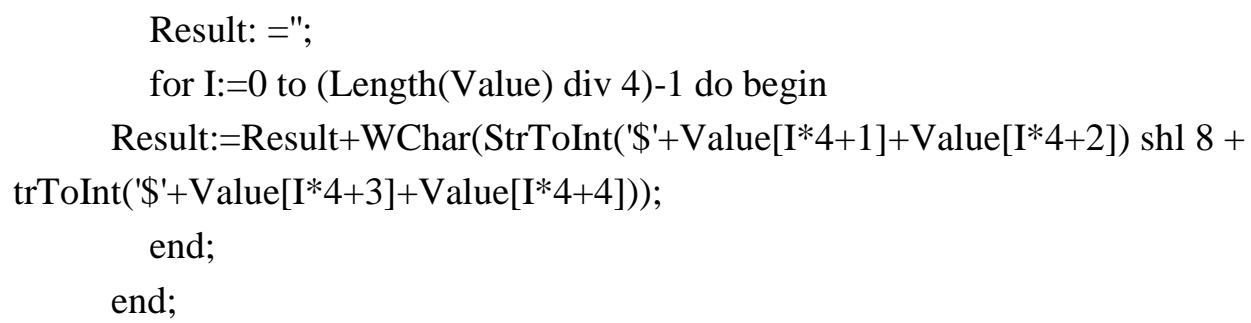

\subsection{The Merits of this System}

The E-government based on OA aggregate the traditional merits and also has its own advantages. The merits are presented in the following aspects.

(1) Betimes. Publics can learn some important information by mobile telephone instead of Internet.

(2) Security. Security is a relative concept. Compared with the security of the Internet, the security level of mobile telephone is higher.

(3) Alternation. This system can feed back the users' information to government to promote the alternation between publics and governments.

\section{Conclusion}

E-government is the decline of the OA. The construction level of E-government affects the whole work efficiency. This paper studies on the OA mode and realize the system. This system can well realize information alternation and betimes. The mode operation is simple, users needn't learn AT instruction sets of GSM mode. The system may help the government to improve the work efficiency and strengthen the relationship between publics and government.

\section{Acknowledgements}

Our work is supported by the National nature science foundation of China (No. 61103161), the Program for New Century Excellent Talents in University (NCET-12-0579) and the "985" special funds in School of information engineering, Minzu university of China.

\section{References}

[1] J. Melitski, "Capacity and e-government performance", Public Performance \& Management Review, vol. 26, no. 4, (2003), pp. 376.

[2] J. Yuan and S. Hong, "The electron governmental affairs network transacts the safe strategy and realization of the system, Computer Application, vol. 24, (2004), pp. 218-211. (In Chinese)

[3] C. Lambrinoudakis et al., "Security requirements for e-government services: a methodological approach for developing a common PKI-based security policy", Computer Communications, vol. 26, (2003), pp. 1873-1883.

[4] Z. Sun and Q. Lu, "The conception and technology of E-government”, Beijing: People's posts and telecommunications publishing house, (2003). 
[5] WAVECOM, "An introduction to the SMS in PDU mode GSM Recommendation phase 2", (2000), vol. 1, pp. 1-18.

[6] European Telecommunications Standards Institute, Digital Cellular Telecommunications System (Phase 2+)", Technical realization of the Short Message Service (SMS): Pointto- Point (PP), (1999).

\section{Authors}

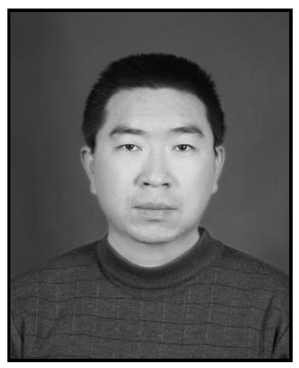

Tao Li. He received his Master Degree in Software Engineering (2007) from Dalian University of Technology. Now he is a lecture of Ludong University at information and electrical engineering department. His current research interests include network technology, artificial intelligence and distributed systems.

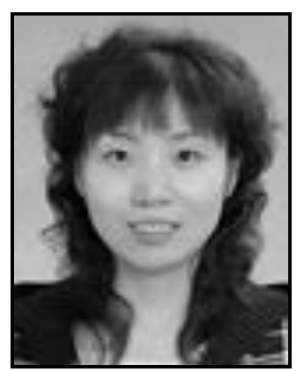

Lirong Qiu. She received her Ph.D. in Computer Sciences (2007) from Chinese Academy of Science. Now she is an associate professor of computer sciences at Information Engineering Department, Minzu University of China. Her current research interests include different aspects of natural language processing, artificial intelligence and distributed systems. 